\title{
A Novel Time Stamping Data Acquisition System Using a Digital Oscilloscope for Single Photon Counting
}

\author{
Myriam Nonaka*, Mónica Agüero, Marcelo Kovalsky and Alejandro Hnilo \\ CEILAP, Centro de Investigaciones en Láseres y Aplicaciones, UNIDEF (MINDEF-CONICET) \\ J.B. de La Salle 4397, (1603) Villa Martelli, Buenos Aires, Argentina \\ *Email: mnonaka@mindef.gob.ar
}

\begin{abstract}
In this paper we present an algorithm of signal processing that allows the use of a PC-based oscilloscope in order to count events with a time stamping register. This novel application has a direct use on recording of arrival times of individual photons detected on one or more detection channels in quantum optics experiments. In particular, we report experimental results of the time stamping acquisition with a temporal resolution of 6.4 ns of non-periodic signals from single-photon counting modules.
\end{abstract}

Index Terms-Signal analysis, Time measurement, Solid lasers, Optical signal detection

\section{INTRODUCCIÓN}

La tecnología de fotones individuales tiene numerosas aplicaciones en ciencia básica y aplicada. Dentro del área de información cuántica algunos ejemplos incluyen distribución cuántica de claves o criptografía cuántica [1], [2], protocolos de teleportación [3] y repetidores cuánticos [4]. Gracias a este tipo de tecnología también fue posible estudiar problemas en el área de fundamentación de la Mecánica Cuántica [5], [6]. Dentro del área de la biología, la detección de fotones únicos permitió realizar grandes avances en el estudio de propiedades de moléculas individuales a partir de técnicas avanzadas de espectroscopía de fluorescencia [7], [8]. Para cada una de estas aplicaciones fue necesario el desarrollo de detectores con una sensibilidad suficientemente alta que registre la llegada de cada uno de los fotones individuales (single-photon detector technologies).

En muchos de estos estudios alcanza con conocer el número de eventos o coincidencias (entre eventos de varios detectores y dentro de una cierta ventana temporal) detectados bajo ciertas condiciones en el experimento. Sin embargo, cuando se quiere estudiar en detalle procesos dinámicos o correlaciones (entre eventos detectados) con resolución temporal es necesario conocer en que instante de tiempo los fotones fueron detectados. Este modo de adquisición conocido como time tagging o time stamping permite generar listas con los tiempos de llegada de los fotones detectados y los datos pueden ser procesados a posteriori, con la libertad de variar a voluntad la ventana de coincidencias, una vez finalizada la medición. Para este modo de adquisición suele emplearse una tarjeta de adquisición de datos o una unidad más sofisticada para el conteo digital de estos eventos (conocidos en inglés como counter/timer devices, time to digital converter, time tagging module) [9]-[11].

En el siguiente trabajo se propone una alternativa accesible para el registro de las listas time stamping con equipamiento de uso general en cualquier laboratorio cuando no se cuenta con una tarjeta de adquisición de datos para conteo de eventos. En particular se presenta un algoritmo de procesamiento de señales que permite emplear un osciloscopio digital para PC para el conteo de fotones, de manera que cada fotón detectado se registre temporalmente como un único evento. Este desarrollo tiene aplicación inmediata en experimentos de óptica cuántica para el estudio de correlaciones con resolución temporal en sistemas de dos fotones en estados entrelazados.

\section{ARREGLO EXPERIMENTAL}

Para la generación de pares de fotones entrelazados en polarización se empleó una de las técnicas más difundidas en el área (figura 1). Estos pares de fotones se producen

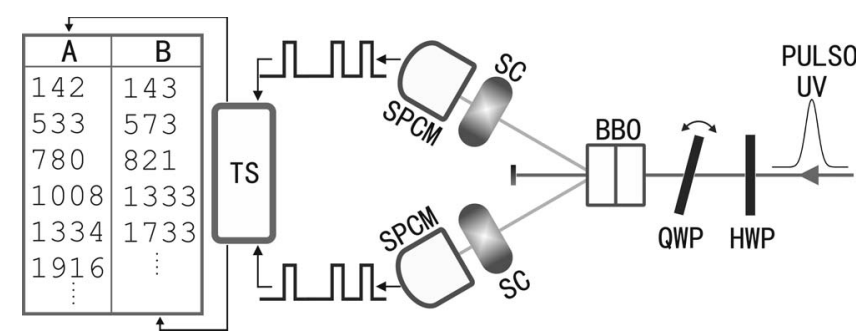

Figura 1. Esquema del dispositivo experimental. HWP: lámina de media onda; QWP: lámina de cuarto de onda; BBO: cristales no lineales; SC: sistema de colección y detección de fotones formado por polarizadores, filtros y objetivos de microscopio que acoplan la radiación a las fibras ópticas conectadas a los detectores (SPCM). TS: registro time stamping. En la tabla se indican el tiempo de detección de cada fotón.

por fluorescencia paramétrica usando un par de cristales no lineales BBO $\left(3 \times 3 \times 1 \mathrm{~mm}^{3}\right.$ cada uno $)$ cortados para phasematching tipo I, con sus ejes ópticos rotados a $90^{\circ}$. El láser de bombeo emite $\approx 4 \mathrm{~mW}$ de radiación linealmente polarizada en $355 \mathrm{~nm}$ a $60 \mathrm{kHz}$ (con una duración de pulso FWHM de 35 ns). Los detalles del arreglo experimental se describen en [12]. Los fotones se detectaron empleando fotodiodos de avalancha de silicio (SPCM - single-photon counting modules) SPCMAQR-13-FC de Perkin-Elmer. El registro time stamping para 
el estudio del grado de entrelazamiento del sistema con resolución temporal se realizó con un osciloscopio digital para PC (PC Oscilloscope PicoScope 6403B) que cuenta con un ancho de banda de $350 \mathrm{MHz}$ y una frecuencia de muestreo máxima de $5 \mathrm{Gs} / \mathrm{s}$.

Notar que en [12] (y en publicaciones anteriores del grupo) el registro time stamping se realizó con una tarjeta de adquisición de datos PCI-6602 de National Instrument con una resolución temporal de 12,5 ns. La propuesta de usar el PicoScope 6403B permite mejorar esta resolución alcanzando los 6,4 ns.

El uso de un osciloscopio digital para PC para conteo de eventos individuales con resolución temporal no es una aplicación estándar de los osciloscopios. En particular, cada módulo SPCM para conteo de fotones emite un pulso eléctrico TTL por cada fotón detectado. La necesidad de desarrollar este algoritmo surge por la gran cantidad de datos con registro time stamping que deben acumularse por períodos largos de medición y con una resolución temporal del orden de unos pocos nanosegundos. Típicamente las mediciones pueden llegar a durar desde varios minutos hasta 1 hora. Dado que sólo interesa el instante de tiempo que indica la llegada de un fotón al detector, si no se contara con el algoritmo de filtrado sería imposible realizar con este instrumento mediciones de estas características.

Para emplear un osciloscopio digital con registro time stamping (ver figura 2) fue necesario desarrollar un algoritmo adicional que cumpla con los siguientes requerimientos:

1- Procesamiento de la señal de entrada de forma que cada pulso TTL se registre como un único evento.

2- Almacenar el instante de tiempo que corresponde a cada evento (time stamping).

3- Resolución temporal menor o igual a 12,5 ns.

4- Procesamiento de la señal en tiempo real.

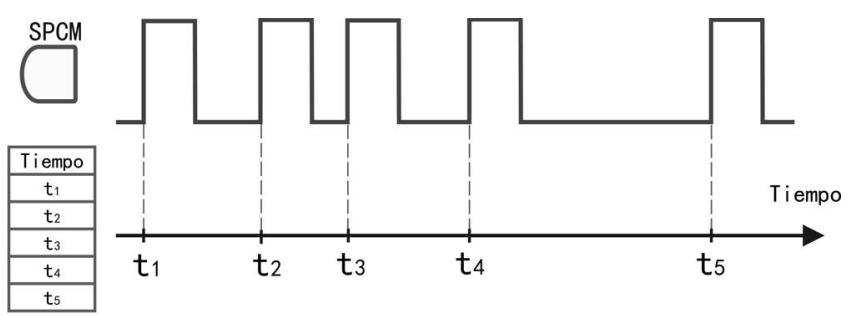

Figura 2. Descripción esquemática de la adquisición de datos time stamping Por cada fotón detectado el SPCM emite un pulso eléctrico TTL. Con time stamping sólo interesa almacenar los tiempos $\mathrm{t}_{1}, \mathrm{t}_{2}, \ldots$ correspondientes a la llegada de cada fotón.

El algoritmo desarrollado se basa en la detección de umbrales de tensión de una señal. El procesamiento de la señal se realizó en Matlab aprovechando los paquetes de software de Matlab incluídos en el PicoScope. En la figura 3 se presenta un diagrama de flujo del algoritmo del software.

La tarea se inicia con la configuración de la frecuencia de muestreo de los datos $\left(f_{a d q}\right)$ y el seteo del umbral de tensión $V_{t h}$ (fijado por el usuario en base a la señal que

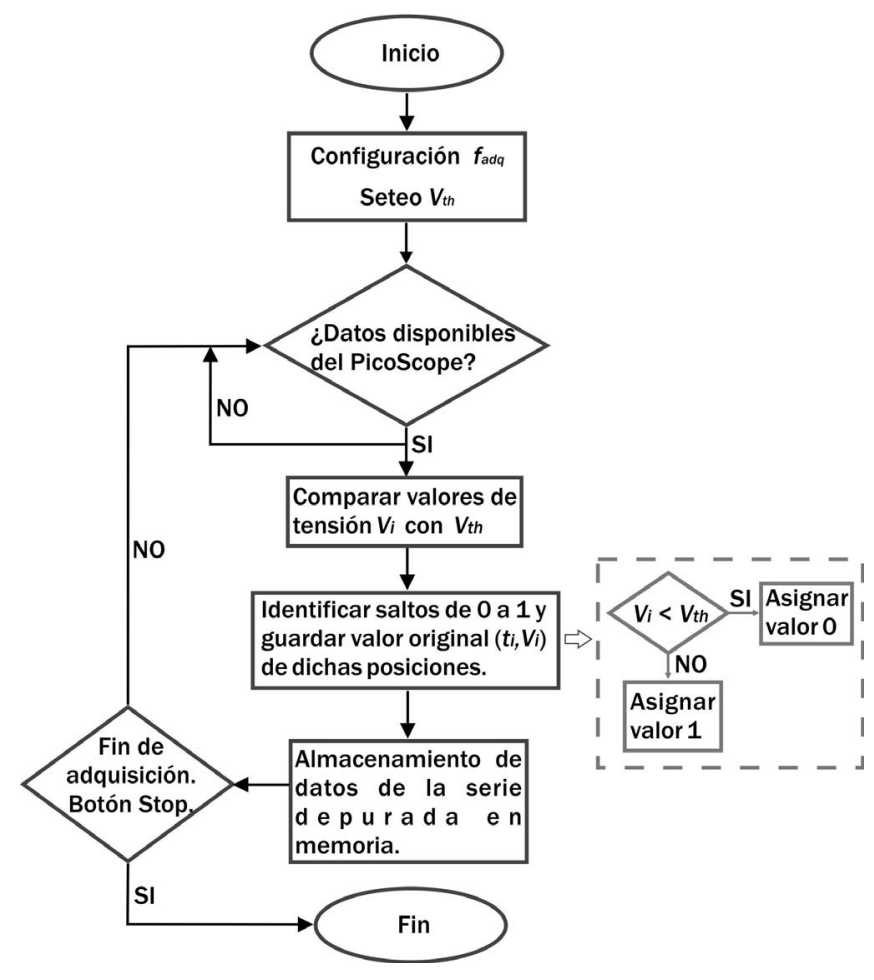

Figura 3. Diagrama de flujo del algoritmo.

se quiera procesar). Además, en esta etapa el PicoScope se configura en el modo streaming. En este modo los datos pasan directamente a la PC sin ser almacenados en la memoria buffer del osciloscopio. Esto permite capturar datos durante un tiempo indefinidamente largo, a alta velocidad y de manera continua, donde el tamaño de la serie está limitado solo por la memoria de la PC. Una vez seteado el modo streaming, la máxima resolución temporal que puede alcanzar el PicoScope es de 6,4 ns.

Los datos registrados por el PicoScope se almacenan por bloques en la memoria de la PC (tamaño máximo del buffer = $2 \mathrm{M}$ ). Notar que este almacenamiento es temporario hasta finalizar el procesamiento del bloque. Aquí se generan dos archivos: uno de ellos contiene la serie original de tensión en función del tiempo $\left(t_{i}, V_{i}\right)$ y el segundo es una copia sobre la cual se realizará la depuración de los datos.

En la etapa siguiente se inicia el procesamiento de la información de cada bloque comparando el valor de tensión medido $V_{i}$ con el valor de tensión umbral. De esta manera se genera un vector auxiliar que se completa con valores 0 y 1 dependiendo del valor de $V_{i}$ en relación con el valor de $V_{t h}$. Si $V_{i}<V_{t h}$, se le asigna el valor 0 . En caso contrario, se le asigna el valor 1 . Luego de convertir la serie a binario se identifican los cambios de valor de 0 a 1 dentro del bloque y se guardan los valores originales $\left(t_{i}, V_{i}\right)$ asociados a dichas posiciones. De esta manera, la serie depurada informa la llegada $t_{i}$ de cada pulso, que se identifica con el primer valor $V_{i}$ (de cada pulso) que supera el $V_{t h}$ elegido.

El algoritmo de prueba guarda la información completa de 
la serie original y la serie depurada. Esto nos permitirá testear la bondad del algoritmo (ver sección III). Sin embargo, el algoritmo que se empleará para el conteo de fotones individuales con time stamping sólo guarda la información temporal de la serie depurada.

\section{IMPLEMENTACIÓN DEL SISTEMA DE ADQUISICIÓN DE DATOS}

A continuación se describe la adaptación del osciloscopio digital para el registro de series de tiempos que indican la llegada de un fotón en el experimento.

Primero se realizó una validación del método a través de una medición simultánea usando el PicoScope y la tarjeta de adquisición de datos PCI-6602. Como se mencionó en la sección II, esta tarjeta de adquisición ha sido empleada con éxito en experimentos anteriores de este tipo [6]. Con un generador de funciones SIGLENT SDG 25MHZ 125MSa/s se generaron pulsos cuadrados a una frecuencia de repetición de $1 \mathrm{kHz}$ que simulan la señal que se quiere filtrar.

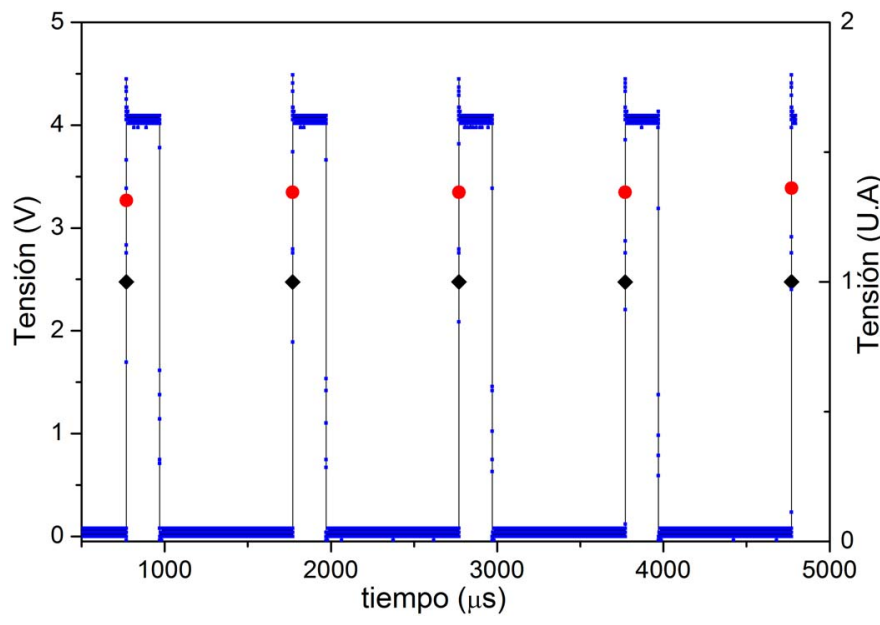

Figura 4. Variación de la tensión en función del tiempo. Con círculos rojos se indican los valores depurados almacenados por el PicoScope mientras que los rombos negros representan los datos adquiridos con la tarjeta PCI-6602.

La señal registrada por el osciloscopio se salva en dos archivos. El primero contiene la información completa de la variación de la tensión en función del tiempo de todos los pulsos y el segundo tiene información de la señal procesada. Esto significa que sólo se almacenan aquellos valores de tiempo y tensión que superan el umbral predefinido por el usuario y que indican la llegada de un pulso. En la figura 4 se muestra la detección de los umbrales de tensión (círculos rojos) de la señal cuadrada indicando que el algoritmo desarrollado detecta de forma correcta la llegada de cada pulso. Además se compararon estos resultados con los obtenidos usando la placa PCI-6602 (rombos negros) para validar el algoritmo desarrollado. En la figura 4 se asignó un valor de tensión en unidades arbitrarias a los datos de la tarjeta PCI-6602 dado que la placa sólo almacena los tiempos que definen la llegada de un pulso.
En todas las pruebas realizadas se observó que los tiempos depurados coinciden con los tiempos registrados por la placa PCI-6602. Esto se hizo verificando que los resultados obtenidos con los sistemas de adquisición sean indistinguibles. Si $t_{\alpha} \pm \Delta t_{\alpha}$ son los tiempos depurados que almacena el PicoScope y $t_{\beta} \pm \Delta t_{\beta}$ son los tiempos que registra la tarjeta PCI-6602, entonces las medidas son indistiguibles si se cumple $\left|t_{\alpha}-t_{\beta}\right| \leq \Delta t_{\alpha}+\Delta t_{\beta}$. Notar que $\Delta t_{\alpha}$ y $\Delta t_{\beta}$ están definidos por la resolución temporal de cada uno de los sistemas de adquisición: 6.4 ns para el PicoScope y 12.5 ns en el caso de la placa PCI-6602. Los resultados obtenidos indican que el el registro time stamping que se mide empleando el PicoScope es el mismo que se obtiene utilizando la placa PCI-6602 pero incrementando la resolución temporal a $6.4 \mathrm{~ns}$.

A continuación se muestra el desempeño del PicoScope adaptado para registro time stamping usando los módulos para conteo de fotones. Con la detección de un fotón, los SPCMAQR emiten un pulso TTL con un duración temporal de 35 ns y un tiempo muerto de $50 \mathrm{~ns}$ entre pulso y pulso. En estos módulos además se producen estadísticamente entre 100 y 250 cuentas de oscuridad por segundo, generadas principalmente por los portadores que se producen vía procesos térmicos o por efecto túnel.

El registro time stamping se realizó empleando el osciloscopio digital PicoScope junto con el algoritmo de procesamiento de datos que se describió en la sección ??. La adquisición se realizó con una resolución temporal de 6,4 ns (máxima resolución temporal en modo streaming). El esquema de adquisición se muestra en la figura 5. A diferencia de los resultados que

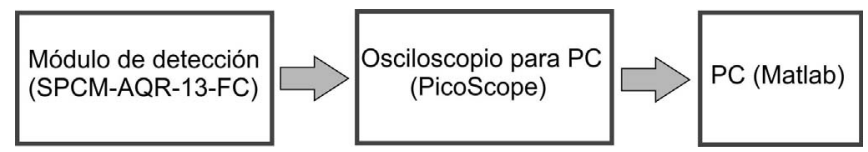

Figura 5. Esquema de adquisición de series temporales experimentales. Cada vez que se detecta un fotón, el módulo de detección envía un pulso TTL al osciloscopio que luego se procesa en la PC.

se muestran en la figura 4, donde los pulsos llegan de manera periódica al osciloscopio, los pares de fotones que se generan en el cristal BBO se producen de forma espontánea. Esto hace que los fotones no lleguen de forma regular a cada uno de los detectores. Además debe tenerse en cuenta que las cuentas de oscuridad también generan pulsos TTL de forma aleatoria.

Como primera prueba en el experimento real se registraron los pulsos TTL de los SPCM con el osciloscopio y se generaron dos archivos. En un archivo se almacenó el registro completo de la señal mientras que en el segundo sólo se guardó la información del valor de tiempo y tensión asociada a la llegada de un fotón. Este último archivo se obtiene una vez procesada la señal con el algoritmo desarrollado en Matlab.

En la figura 6(a) se muestra la adquisición de una serie temporal experimental donde se puede observar los pulsos provenientes del módulo de detección (que no llegan de forma regular) y sus respectivos umbrales marcados con un círculo. En la figura 6(b) se muestra la señal ampliada correspondiente a un único pulso del SPCM donde se observa en detalle la 
forma del pulso que emiten los detectores y la correspondiente detección para el registro time stamping. Además se

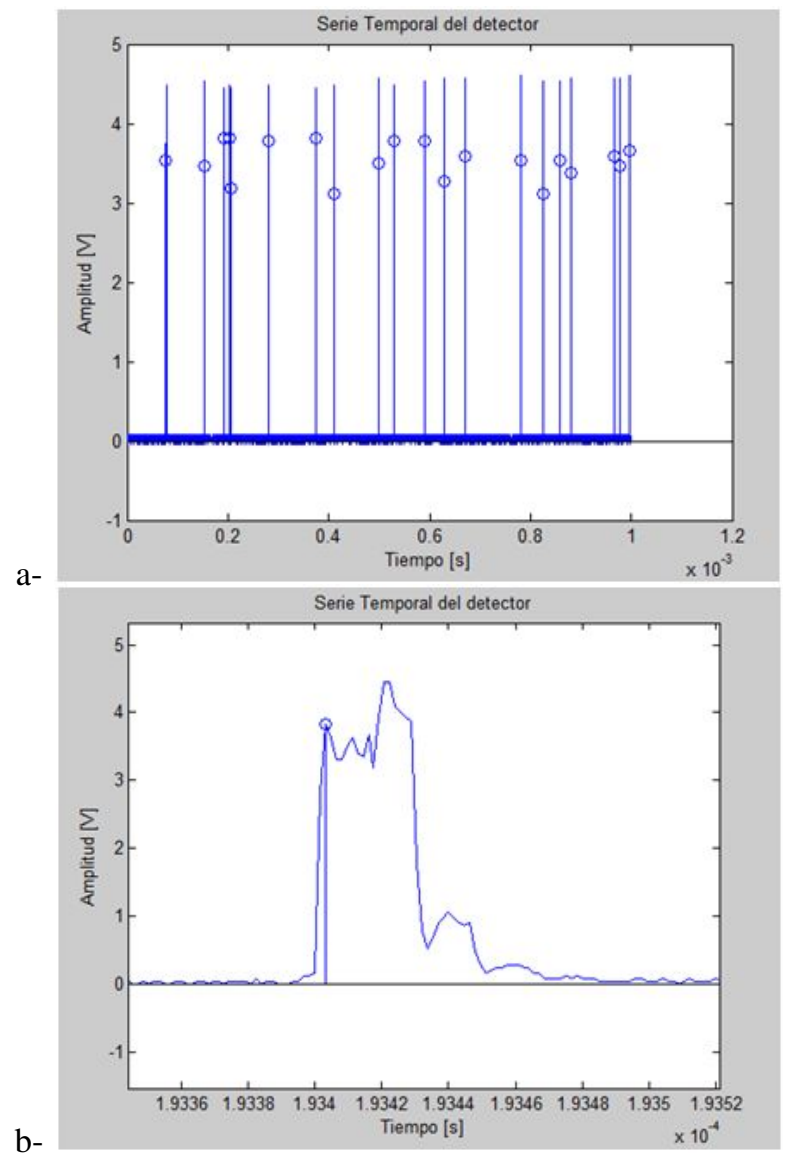

Figura 6. a- Serie temporal experimental. b- Detalle de la imagen (a) donde se observa la forma del pulso que el SPCM genera con cada detección. Con un círculo se indica el registro time stamping.

verificó que, aún cuando los pulsos no se generan de forma periódica, las listas de tiempos que registran el PicoScope y la placa PCI-6602 son indistiguibles (empleando el criterio que se describió al principio de esta sección); con la ventaja de alcanzar una mayor resolución temporal cuando se emplea el PicoScope.

\section{CONCLUSión}

En este trabajo se presentó una aplicación novedosa en el uso de un osciloscopio digital para PC para el conteo de eventos con resolución temporal. A partir del procesamiento de las señales de los módulos SPCM para conteo de fotones se desarrolló un algoritmo que permitió usar un osciloscopio digital en el registro de series time stamping. Esta adquisición se realiza en tiempo real y con una resolución temporal máxima de 6,4 ns. Esto representa una mejora en el experimento descripto en la sección II en relación con el sistema de adquisición previamente usado (máxima resolución alcanzada: $12,5 \mathrm{~ns}$ ). Sin embargo, aunque otras empresas (ver [10], [11]) ofrecen unidades más sofisticadas con una resolución temporal de picosegundos, para muchas aplicaciones donde sólo se requiere una precisión de unos pocos nanosegundos, el algoritmo propuesto es de gran utilidad si se evalúa la relación costo - beneficio.

\section{AGRADECIMIENTOS}

Este trabajo fue realizado con los aportes de los subsidios PIP2011-077 del CONICET y PIDDEF 18/14 del Ministerio de Defensa de la República Argentina.

\section{REFERENCIAS}

[1] A. Ekert, "Quantum cryptography based on Bell's theorem", Phys. Rev. Lett. 67, 661 (1991).

[2] T. Jennewein, C. Simon, G. Weihs, H. Weinfurter, and A. Zeilinger, "Quantum Cryptography With Entangled Photons", Phys. Rev. Lett. 84, 4729 (2000).

[3] C. Bennett, G. Brassard, C. Crepeau, R. Jozsa, A. Peres, and W. Wootters,"Teleporting an unknown quantum state via dual classical and Einstein-Podolsky-Rosen channels", Phys. Rev. Lett. 70, 1895 (1993).

[4] N. Sangouard, C. Simon, B. Zhao, Y. Chen, H. de Riedmatten, J. Pan, and N. Gisin, "Robust and efficient quantum repeaters with atomic ensembles and linear optics",Phys. Rev. A 77, 062301 (2008).

[5] M. Giustina, A. Mech, S. Remelow, B. Wittmann, J. Kofler, J. Beyer, A. Lita, Calkins B., T. Gerrits, S. Woo Nam, R. Ursin, and A. Zeilinger, "Bell violation using entangled photons without the fair-sampling assumption", Nature 497, 227 (2013).

[6] M. Agüero, A. Hnilo, and M. Kovalsky, "Time-resolved measurement of Bell inequalities and the coincidence loophole", Physc. Rev. A 86, 052121 (2012).

[7] M. Wahl, I. Gregor, M. Patting, and J. Enderlein, "Fast calculation of fluorescence correlation data with asynchronous time-correlated singlephoton counting”, Opt. Express 11, 3583 (2003).

[8] G. Persson, P. Thyberg, and J. Widengren, "Modulated Fluorescence Correlation Spectroscopy with Complete Time Range Information", Biophys. J. 94, 977 (2008).

[9] M. Agüero, A. Hnilo, M. Kovalsky, M. Larotonda, "Time stamping in EPRB experiments: application on the test of non-ergodic theories", Eur. Phys. J. D 55, 705 (2009).

[10] Time Tagging Module: www.roithner-laser.com/scientific.html

[11] Time-Correlated Single Photon Counting and Time Tagging Electronics: www.picoquant.com/products/category/tcspc-and-time-tagging-modules

[12] M. Agüero, A. Hnilo, and M. Kovalsky, "Measuring the entanglement of photons produced by a nanosecond pulsed source", J. Opt. Soc. Am. B 31, 3088 (2014) 